\title{
Obsessive Compulsive Phenomenon: Measurement of Normal Obsessions in a Non-Clinical Female Population
}

\author{
Pragya Lodha ${ }^{1}$, Deepika Bhandari ${ }^{2}$ \\ ${ }^{1}$ T.Y.B.A. student of Psychology, Sophia College for Women, Mumbai. \\ ${ }^{2}$ T.Y.B.A. student of Psychology, Sophia College for Women, Mumbai. \\ E-mail - pragya6lodha@gmail.com
}

\begin{abstract}
Introduction: Obsessive Compulsive Disorder (OCD) is the fourth most common psychological disorder; and epidemiological studies have shown that OCD has a prevalence rate of about $2 \%$. Normal obsessions are a common experience that are similar to abnormal obsessions, in their form and content and researchers propose the existence of a continuum between normal intrusive thoughts and clinical obsessions which enable the study of obsessive phenomenon in non-clinical populations.

Methodology: The research undertaken was exploratory in nature and done to observe the occurrence of normal (non-clinical) obsessions in an undergraduate, sub-clinical female population. The data was obtained by administering a self-report measure of obsessive compulsive symptoms, the Padua Inventory (PI). A randomized sample of 134 female undergraduates, (age range from 18 years to 21 years) was used as the sample for the study from two colleges in Mumbai. Participants were asked to report their age and religion. Scores on the Padua Inventory were tabulated and calculated by simple addition. Separate mean and standard deviation scores on the basis of religion (Hindu, Christian, Muslim and other religions) were calculated to study the effect of religion on occurrence of normal obsession, if any.

Results: The mean score was found to be highest for the Muslim sub-clinical sample, $79.44(\mathrm{SD}=25.21)$. However, the lowest mean score was calculated for the Christian sub-clinical sample, $64.94(\mathrm{SD}=25.73)$. Hindus and Muslims have ritualistic practices that are ingrained in them since childhood, as a consequence of being imposed upon them. Practices like washing hands and feet before sitting for a meal, bathing before entering the temple or the kitchen, washing hands before performing any religious activity, entering a new place or house with the right foot and several other practices, incorporated continually have become habitual.

Conclusions: Results showed that normal obsessions occur to a great extent in the general (non-clinical) population and are mostly overlooked or left unattended. With over 50 participants scoring above the criterion score of 80 (as mentioned), it shows that the OC phenomena is considerably prevalent and can be explored further. The future implications for the study are immense, since such a research has not been undertaken in the Indian context. Such a study can be repeated with males so as to obtain data representative of the entire population and further enable understanding of gender differences in the $\mathrm{OC}$ phenomenon.
\end{abstract}

Key Words: Obsessive Compulsive Disorder (OCD), normal (non-clinical) obsessions, abnormal (clinical) obsessions, Padua Inventory

(Paper received $-14^{\text {th }}$ November 2016, Peer review completed $-4^{\text {th }}$ December 2017, Accepted $-6^{\text {th }}$ December 2016)

\section{INTRODUCTION}

Obsessive Compulsive Disorder (OCD) was described as early as the seventeenth century, the Oxford Don, Robert Burton, reported a case in his compendium, 'The Anatomy of Melancholy' [1]. Obsessive Compulsive Disorder now is among the most common disorders, with disability in severe cases-often comparable to the disability associated with mental disorders such schizophrenia and bipolar disorder [2]. 
It is a neuropsychiatric disorder that involves recurrent and unwanted thoughts, ideas, beliefs or images (obsessions); and uncontrollable urges and seemingly purposeful behavior performed in order to reduce the anxiety caused by obsessions [3]. OCD remains to be an often undiagnosed psychiatric illness, with a poor prognosis, to add on to its incapacitating nature. The disorder is constituted by either or both components of recurrent obsessions and compulsions that cause significant impairment in an individual's daily functioning [4]. There are generally no cultural differences found in the development OCD and nor are there differences based on gender; however the age at which males and females are most likely to develop the disorder varies, with males likely to develop between 6-15 years and females between 20-29 years [5]. Epidemiological studies have shown that the prevalence rate of OCD among young population worldwide ranges from $1-2 \%[6]$.

Obsessions are defined as (1) recurrent and persistent thoughts, urges, or impulses that are experienced, at some time during the disturbance, as intrusive and unwanted, and that in most individuals cause marked anxiety or distress and /or (2) the individual attempts to ignore or suppress such thoughts, urges, or images, or to neutralize them with some other thought or action (i.e. by performing a compulsion). However, even the perpetual effort of the individual to curb these obsessions fails [7].

Individuals with OCD frequently have additional psychiatric disorders concomitantly or at some time during their lifetime [8]. Some authors proposed an OCD sub-classification based on comorbidity which has three classes solution characterized by: (1) an OCD simplex class, in which Major Depressive Disorder (MDD) is the most frequent additional disorder; (2) an OCD comorbid Tic-related class, in which tics are prominent and affective syndromes are considerably rarer; and (3) an OCD comorbid affective-related class in which Panic Disorder (PD) and affective syndromes are highly presented [9].

Operationally, normal obsessions can be defined as recurrent, normal intrusive thoughts that occur to individuals without disrupting the daily functioning of the individuals; whereas abnormal obsessions are repetitive, more intrusive and longer lasting, more insistent and more adhesive thoughts that occur to individuals and cause considerable amount of distress and impairment that is of clinical nature and significance. Normal obsessions are similar to abnormal obsessions in form and content [10]. Several research papers have tended to focus on the obsessional thoughts in normal populations and treatment outcome studies with the clinical populations [11].

Subclinical OC tendencies have been proposed to be another dimension of OCD; that is, individuals who do not meet the diagnostic criteria of the disorder but still are vulnerable to neuropsychological deficits [12]. Today, approximately 10 percent of the population has sub-clinical OCD (where the thoughts are intrusive, but do not impair daily life and ability to function); and almost 12.5 percent of the population experiences OCD symptoms at some point in their lives [13]. Over 12 million Indians are thought to have one or the other form of OCD, about $40 \%$ recover and $30 \%$ show fair improvement [14]. The form and to some extent, the content of non-clinical (normal) obsessions, reported by non-psychiatric respondents is similar to that of clinical (abnormal) obsessions, reported by obsessional patients [15]. The 'normal' obsessions are also similar to 'abnormal' obsessions in their expressed relation to mood and in their meaningfulness to the respondent. Conclusively, normal and abnormal obsessions are similar in form and content; but differ in frequency, intensity and their consequence [16].

\section{METHODOLOGY}

The research undertaken was exploratory in nature. It was done to observe the occurrence of normal (nonclinical) obsessions in an undergraduate, sub-clinical female population. The data was obtained by administering a self-report measure of obsessive compulsive symptoms, the Padua Inventory (PI). A randomised sample of 134 female undergraduates, (age range from 18 years to 21 years) was used as the sample for the study from two colleges in Mumbai. One was a South Bombay (Mumbai) college while the other was located in suburban Mumbai (Juhu). The colleges were chosen to include diversity of population across the two regions of Mumbai. The decision to use only a female population for the study was based on an extended exploration of the gender difference in the occurrence of normal obsessions. Female undergraduates (18 years to 21 years) were chosen to be participants for the study, taking on the crucial age when obsessions are heightened and expressed prominently as compulsions. 
The tool used for the study was the Padua Inventory [17] which is a 60 statement, 5 point for the selfreport measure of symptoms of obsessive compulsive disorder. It uses rating from $0-4$ ranging from 'not at all' to 'very much'. Participants are required to state the degree to which the statement is applicable to them, that is, give them a rating. On the basis of these ratings, a score is obtained. Scores were calculated by simple addition. The scores can range from $0-240$. The scale lays down a criterion score of 80 . If the participant's score obtained exceeds this value, he/she fits into the criterion for clinical intervention to deal with Obsessive Compulsive Disorder (OCD).

The data was collected by means of a survey administered manually. Participants were asked to report their age and religion, however anonymity was maintained. This aided in avoiding apprehensions and allowed for accurate and honest responses. Scores on the Padua Inventory were tabulated and calculated by simple addition. The mean score and standard deviation for the total population was obtained. Separate mean and standard deviation scores on the basis of religion (Hindu, Christian, Muslim and other religions) were calculated to study the effect of religion on occurrence of normal obsession, if any.

\section{RESULTS}

The mean score for the sample was calculated to be 75.18 , as shown in Table 1 . The lowest score obtained was 17 , while the highest score obtained was 137 . The consequent range was thus found to be 120 . The standard deviation was calculated and the value obtained was 24.77 , rounded off to two decimal values.

Table 1: Mean and Standard Deviation of the Sample

\begin{tabular}{|ccc|}
\hline Sample Size & Mean & Standard Deviation \\
\hline 134 & 75.18 & 27.77 \\
\hline
\end{tabular}

The mean and standard deviation scores calculated have been tabulated in Table 2. The mean score was found to be highest for the Muslim sub-clinical sample, 79.44 (SD = 25.21). However, the lowest mean score was calculated for the Christian sub-clinical sample, $64.94(\mathrm{SD}=25.73)$. The mean scores obtained are possibly not representative of the entire group population due to inadequate sampling (16 in each category). To test the significance of religion, culture and religious practices, on the prevalence of normal obsessions and compulsions, a one way ANOVA was performed. The results obtained were found to be not significant at the alpha level of 0.05 . This result can be attributed to negligible difference in the mean scores for the Muslim and Hindu sub-clinical samples (difference in mean $=2.31$ ) which was found to be not significant.

Table 2 - Mean and Standard deviation of Religious Samples

\begin{tabular}{|cccc|}
\hline Sample Size & Religion & Mean & $\begin{array}{c}\text { Standard } \\
\text { Deviation }\end{array}$ \\
\hline 84 & Hindu & 77.31 & 29.65 \\
\hline 16 & Muslim & 79.44 & 25.21 \\
\hline 16 & Christian & 64.94 & 25.73 \\
\hline 18 & Other Religions & 70.56 & 20.81 \\
\hline
\end{tabular}

It was observed that the variation of the mean score of Christian sub-clinical sample from both the Hindu and the Muslim sub-clinical sample was large. To test for significance of this difference, a t-test was performed individually on the Christian and Hindu; and the Christian and Muslim sub-clinical samples. The t-values calculated have been tabulated in Table 3. In both instances, the obtained value was found to be significant at the alpha level of 0.01 . These results are indicative of the importance of religion and religious practice that play a key role in determining obsessive thoughts and/or compulsive behaviours. 
Table 3 - Difference in Mean and t-value

\begin{tabular}{|ccc|}
\hline Sample Groups & Difference in Mean & $\mathrm{t}$-value \\
\hline Hindu-Christian & 12.37 & $\mathrm{t}=2.81^{*}$ \\
\hline Muslim-Christian & 14.5 & $\mathrm{t}=2.75^{*}$ \\
\hline \multicolumn{3}{|c}{ *significant at $\mathrm{p}<0.01$; one-tailed }
\end{tabular}

The obtained results can be explained as a function of culture and its underlying influence on the upbringing of individuals and thus is reflective on their diurnal chores. Hindus and Muslims have ritualistic practices that are ingrained in them since childhood, as a consequence of being imposed upon them. Practices like washing hands and feet before sitting for a meal, bathing before entering the temple or the kitchen, washing hands before performing any religious activity, entering a new place or house with the right foot and several other practices, incorporated continually have become habitual. This habituation reaches a level that the individual starts performing them without conscious thought.

These imposed activities later progress to be expressed as normal obsessions and a person feels uncomfortable when not allowed to perform them freely. In case of Christian practice however, these customary performances are not imposed to such a great extent which may be the cause for the significant difference obtained. Alternatively, these differences may have been obtained as a result of unequal subclinical sample size and additionally, because the Hindu sub-clinical sample was relatively more representative than the Muslim and Christian sub-clinical sample.

As an ancillary observation, a t-test with a significant $t$-value at the alpha level of 0.01 was noted and recorded for the Muslim and Christian sub-clinical samples. These results are suggestive that the results so found are due to the factor religion (since the sample size for both was equal).

\section{DISCUSSION}

The mean and standard deviation scores calculated have been tabulated in Table 2. The mean score was found to be highest for the Muslim sub-clinical sample, $79.44(\mathrm{SD}=25.21)$. However, the lowest mean score was calculated for the Christian sub-clinical sample, $64.94(\mathrm{SD}=25.73)$. The mean scores obtained are possibly not representative of the entire group population due to inadequate sampling (16 in each category). To test the significance of religion, culture and religious practices, on the prevalence of normal obsessions and compulsions, a one way ANOVA was performed. The results obtained were found to be not significant at the alpha level of 0.05 . This result can be attributed to negligible difference in the mean scores for the Muslim and Hindu sub-clinical samples (difference in mean $=2.31$ ) which was found to be not significant.

It was observed that the variation of the mean score of Christian sub-clinical sample from both the Hindu and the Muslim sub-clinical sample was large. To test for significance of this difference, a t-test was performed individually on the Christian and Hindu; and the Christian and Muslim sub-clinical samples. The t-values calculated have been tabulated in Table 3. In both instances, the obtained value was found to be significant at the alpha level of 0.01 . These results are indicative of the importance of religion and religious practice that play a key role in determining obsessive thoughts and/or compulsive behaviours.

The obtained results can be explained as a function of culture and its underlying influence on the upbringing of individuals and thus is reflective on their diurnal chores. Hindus and Muslims have ritualistic practices that are ingrained in them since childhood, as a consequence of being imposed upon them. Practices like washing hands and feet before sitting for a meal, bathing before entering the temple or the kitchen, washing hands before performing any religious activity, entering a new place or house with the right foot and several other practices, incorporated continually have become habitual. This habituation reaches a level that the individual starts performing them without conscious thought. These imposed activities later progress to be expressed as normal obsessions and a person feels uncomfortable when not allowed to perform them freely. In case of Christian practice however, these customary performances are 
not imposed to such a great extent which may be the cause for the significant difference obtained. Alternatively, these differences may have been obtained as a result of unequal sub-clinical sample size and additionally, because the Hindu sub-clinical sample was relatively more representative than the Muslim and Christian sub-clinical sample.

As an ancillary observation, a t-test with a significant t-value at the alpha level of 0.01 was noted and recorded for the Muslim and Christian sub-clinical samples. These results are suggestive that the results so found are due to the factor religion (since the sample size for both was equal).

The future implications for the study are immense, since such a research has not been undertaken in the Indian context. Such a study can be repeated with males so as to obtain data representative of the entire population and make required comparisons. This would also facilitate understanding of differences in the nature of normal obsessions between the genders. The population tested could have been better represented which would yield results that could be used as normative data for future tests of normal or abnormal obsessions and compulsions for the chosen cohort of population.

The possibility of extending the study to different age groups to facilitate greater and a more wholesome understanding can be considered. The specified age groups could be: young adults (21-35), older adults (35-60) and senior citizens (60 onwards), each with a sample representative of both genders and of the population. Norms could be laid down for the same. In addition, a separate study could be undertaken to understand the nature of obsessions and compulsions separately, since obsessions are persistent thought processes while compulsions are persistent behavioural patterns.

\section{CONCLUSIONS}

Results showed that normal obsessions occur to a great extent in the general (non-clinical) population and are mostly overlooked or left unattended. With over 50 participants scoring above the criterion score of 80 (as mentioned), it shows that the OC phenomena is considerably prevalent and can be explored further. A limitation encountered in the study is with regard to the sample size and sample type of the population because the chosen sample was not representative of the entire population, henceforth normative data could not be obtained. Randomized sampling, if chosen instead, could have adequately been representative of the population, enabling a clearer understanding of the occurrences of obsessions and compulsions.

\section{REFERENCES}

1. Burton R. 1621. The anatomy of melancholy. Oxford ; 1927.

2. Weissman MM. Cross-national epidemiology of obsessive-compulsive disorder. CNS Spectr 1998;3(S1):6-9.

3. Angst J, Gamma A, Endrass J, Goodwin R, Ajdacic V, Eich D, Rössler W. Obsessive-compulsive severity spectrum in the community: prevalence, comorbidity, and course. Eur Arch Psychiatr Clin Neurosci 2004;254(3):156-64.

4. Karno M, Golding JM, Sorenson SB, Burnam MA. The epidemiology of obsessive-compulsive disorder in five US communities. Arch Gen Psychiatry 1988;45(12):1094-9.

5. Horwath E, Weissman MM. The epidemiology and cross-national presentation of obsessive-compulsive disorder. Psychiatr Clin North Am 2000;23(3):493-507.

6. Abramowitz JS, Taylor S, McKay D. Obsessive-compulsive disorder. Lancet 2009 Aug 14;374(9688):491-9.

7. Jenike MA. Obsessive-compulsive disorder. New Eng J Med 2004;350(3):259-65.

8. Angst J, Gamma A, Endrass J, Hantouche E, Goodwin R, Ajdacic V, Eich D, Rössler W. Obsessivecompulsive syndromes and disorders. Eur Arch Psychiatr Clin Neurosci 2005;255(1):65-71.

9. Nestadt G, Di CZ, Riddle MA, Grados MA, Greenberg BD, Fyer AJ, McCracken JT, Rauch SL, Murphy DL, Rasmussen SA, Cullen B. Obsessive-compulsive disorder: subclassification based on co-morbidity. Psychol Med 2009;39(09):1491-501.

10. Shafran R, Thordarson DS, Rachman S. Thought-action fusion in obsessive compulsive disorder. J Anxiety Disord 1996;10(5):379-91.

11. Rachman S. A cognitive theory of obsessions. Behav Res Ther 1997;35(9):793-802. 
12. Grabe HJ, Meyer C, Hapke U, Rumpf HJ, Freyberger HJ, Dilling H, John U. Lifetime-comorbidity of obsessive-compulsive disorder and subclinical obsessive-compulsive disorder in Northern Germany. Eur Arch Psychiatr Clin Neurosci 2001;251(3):130-5.

13. Apter A, Fallon TJ, King RA, Ratzoni G, Zohar AH, Binder M, Weizman A, Leckman JF, Pauls DL, Kron S, Cohen DJ. Obsessive-compulsive characteristics: from symptoms to syndrome. J Am Acad Child Adolesc Psychiatry 1996;35(7):907-12.

14. Jaisoorya TS, Reddy YJ, Srinath S, Thennarasu K. Obsessive-compulsive disorder with and without tic disorder: a comparative study from India. CNS Spectr 2008;13(08):705-11.

15. Srivastava S, Bhatia MS, Thawani R, Jhanjee A. Quality of life in patients with obsessive compulsive disorder: A longitudinal study from India. Asian J Psychiatry 2011;4(3):178-82.

16. Kalra H, Trivedi JK, Dalal PK, Sinha PK, Allet JL. Uncomplicated and complicated obsessive-compulsive disorder: an exploratory study from India. Compr Psychiatry 2008;49(1):51-4.

17. Sanavio E. Obsessions and compulsions: the Padua Inventory. Behav Res Ther 1988;26(2):169-77.

Acknowledgements - Dr. Jennie Mendes, Associate Professor, Department of Psychology, Sophia College for Women, Mumbai

Source of Funding - Nil

Conflict of Interest - Nil 